\title{
Desktop-Based Computer-Assisted Orthopedic Training System for Spinal Surgery
}

\author{
Rohit Rambani, FRCS (Tr \& Orth), ${ }^{*}+$ James Ward, BSc, \\ and Warren Viant, $B S c^{*}$
}

\section{*Department of Computer Sciences, University of Hull, Hull, United Kingdom; and 'Department of Orthopaedics, Leeds Teaching Hospital NHS Trust, Leeds, United Kingdom}

\begin{abstract}
BACKGROUND: Simulation and surgical training has moved on since its inception during the end of the last century. The trainees are getting more exposed to computers and laboratory training in different subspecialties. More needs to be done in orthopedic simulation in spinal surgery.
\end{abstract}

AIMS: To develop a training system for pedicle screw fixation and validate its effectiveness in a cohort of junior orthopedic trainees.

TRAINING SYSTEM: Fully simulated computer-navigated training system is used to train junior orthopedic trainees perform pedicle screw insertion in the lumbar spine. Real patient computed tomography scans are used to produce the real-time fluoroscopic images of the lumbar spine.

MATERIAL AND METHODS: The training system was developed to simulate pedicle screw insertion in the lumbar spine. A total of 12 orthopedic senior house officers performed pedicle screw insertion in the lumbar spine before and after the training on training system. The results were assessed based on the scoring system, which included the amount of time taken, accuracy of pedicle screw insertion, and the number of exposures requested to complete the procedure.

RESULTS: The result shows a significant improvement in amount of time taken, accuracy of fixation, and the number of exposures after the training on simulator system. This was statistically significant using paired Student $t$ test $(\mathrm{p}<0.05)$.

CONCLUSION: Fully simulated computer-navigated training system is an efficient training tool for young orthopedic trainees. This system can be used to augment training in the

Correspondence: Inquiries to Rohit Rambani, MBBS, MSortho, MRCSEng, FIMSA, FRCS (Tr \& Orth), House no. 82, Mackenzie Crescent, Sheffield S35 1US, UK; e-mail: rohit@rambani.com operating room, and trainees acquire their skills in the comfort of their study room or in the training room in the hospital. The system has the potential to be used in various other orthopedic procedures for learning of technical skills in a manner aimed at ensuring a smooth escalation in task complexity leading to the better performance of procedures in the operating theater. ( $\mathrm{J}$ Surg 71:805-809. ( 2014 Association of Program Directors in Surgery. Published by Elsevier Inc. All rights reserved.)

KEY WORDS: training system, spinal fractures, computer navigation, simulation

COMPETENCIES: Practice-Based Learning and Improvement, Systems-Based Practice, Medical Knowledge

\section{INTRODUCTION}

Surgical training has progressed from the era of doing the training on the plastic bone simulator to 3-dimensional (3D) simulation using special monitors and cameras. ${ }^{1}$ As surgeons, the primary goal of our training is to provide the best care to our patients. However, there is always a scope for improvement in surgeon education and training, and simulation can provide a platform for solving these training riddles. ${ }^{2}$ More and more orthopedic training programs are making the simulation training a mandatory part of the training. The challenges of reduced training opportunities, shortened working hours, and financial pressures ${ }^{3}$ have been exemplified by the increased pressures on the hospitals to achieve targets and focus on service provision. Trainees are developing their psychomotor skills in their later years of training, and highly specialized surgeries like spinal surgery are mainly done by the consultants or the fellows who have decided on their subspecialty. ${ }^{4}$ Training simulators are available to the selected few, and most trainees have to register to courses and workshops to do simulation for the specific procedures. Simulation systems provides stress-free 
environment for junior trainees to learn surgical skills. ${ }^{5}$ Simulations are used to augment training in the operating room, and trainees acquire their skills in structured manner aimed at ensuring a smooth escalation in task complexity to improve performance in the theater. ${ }^{6}$ It also gives an opportunity for the trainers to assess the trainees on simulator before they can perform the surgery on the actual patients.

Intraoperative fluoroscopy is the tool for navigating the surgeon in the 3-D space using 2-D images. Orthopedic surgeons are accustomed to using this 2-D image data to navigate the surgical instruments in a 3-D space. This is commonly called as the mental navigation, similar to aeronautical navigation. The use of fluoroscopy is of paramount importance in the spinal surgery where the surgeon is working close to the spinal cord and a single mistake can lead to catastrophic complications. Though senior surgeons are used to doing complex procedures without much difficulty, it becomes a daunting task for the junior trainees to learn this skill in the operating theater.

Computer-aided surgery has been used extensively in recent years in various surgical disciplines including the spinal surgery. ${ }^{7-9}$ Most of the research has been done in the past on the use of computer navigation in performing surgery. ${ }^{1,6,9}$ The use of simulation training is still in its infancy when it comes to training in spinal surgery. The procedure for pedicle screw placement is complex and technically demanding with a steep learning curve. ${ }^{10,11}$ The present study tries to test the usefulness of computer-assisted orthopedic training system in the development of mental navigation of the surgical trainee in 3-D space for the common spinal procedure like pedicle screw fixation. The study is initially used to train orthopedic trainees in placement of pedicle screw fixation and see the improvement in their performance after training on computer-assisted orthopedic training system.

\section{Computer-Assisted Orthopedic Training System}

We use the computer-assisted orthopedic system developed by the Simulation and Visualization Research Group, of which earlier versions have been in use since $1992 .{ }^{1,12,13}$ The system has been modified since its initial development to be the desktop system with no use of the fluoroscope and the saw bones. The computed tomography (CT) scan image is loaded into the system, and it produces real-time fluoroscopic images. Using polaris tracking system, computerassisted orthopedic system allows real-time image-interactive navigation of the surgical tools with respect to the 2 preacquired radiographic images processed from the CT scan. The system does not need to acquire any radiographic images during the surgery, and the images are generated by the computer-assisted orthopedic training system from the CT scan. The computer also allows noncontact measurement of precise angles and depth of surgical tool penetration of bone.

\section{Use of Computer-Assisted Orthopedic Training System in the Training of Pedicle Screw Insertion in the Lumbar Spine}

Pedicle screw insertion is one of the most commonly performed steps in the spinal surgery and spinal fracture fixation. Orthopedic trainees learn to do this procedure much later in their training career, and simulation with feedback for this procedure is hardly available.

Computer-assisted orthopedic training system uses CT scan-generated images to register the bone and landmarks. The system has an admin mode where the trainer can put the desired CT scans onto the system. The system identifies the bony cortices and generates 2 image (anteroposterior and lateral) views for the trainer to confirm the landmarks on the bone. The trainer in the admin mode of the system analyzes these images and decides the desired trajectory of the screw. The computer-assisted orthopedic training system guides the trainee to correct orientation and position in the training mode. The assessment mode assesses the performance of the trainee and gives the scores based on the scoring system.

\section{MATERIAL AND METHODS}

The study was designed to include junior orthopedic trainees from the local hospital. All the junior surgical trainees who had not done any spinal surgery and had no previous exposure to computer-assisted orthopedic training system were included in the study. Our hypothesis is that the group that was exposed to computer-assisted orthopedic training would have the same performance as the group that was not exposed to computer-assisted orthopedic training.

The trainees were randomly divided into 2 groups.

\section{Group 1 (Who Had Navigation Training)}

Initial part of the study involved the use of conventional computer-assisted orthopedic surgery system to train this group of orthopedic trainees. This group was trained using the computer-assisted orthopedic surgeries system for pedicle screw insertion in the lumbar spine (6 Trainees).

\section{Group 2 (With No Navigation Training)}

This group would have no exposure to such training (6 Trainees).

The control group was made of equal number of trained orthopedic surgeons who routinely do spinal surgery so that the scoring system can be validated and the 2 groups can be compared with the control group.

The 2 groups are also compared for the difference of exposures required to perform the task and amount of time required to finish the task. 


\section{Power Calculation}

Power calculation was done based on the $80 \%$ power to detect an odds ratio of 5 at a significance level of $5 \%$.

\section{Scoring System}

The ability for precision, 3-D navigation, and processing of virtual information to help in hand-eye coordination have never been used as a formal training tool. The assessment of such skills demands a scoring system, which can be reproducible as well as validated. There is no scoring system that can accurately assess the ability to navigate instruments in 3-D space using a C-arm image.

We modified the previous scoring system based on task analysis that we had used in our previous study ${ }^{1}$; this included time difference between each exposure, the change in 3-D coordinates with each exposure, and total number of exposures to finish the task.

The procedure was broadly divided into 2 tasks:

1. Navigation in 3-D space.

2. Inserting the pedicle screw at the desired position.

These 2 tasks were further divided into smaller subtasks, and the candidate was scored in all of these subtasks. The candidates were assessed using various parameters, which included the following:

\section{3-Dimensional Navigation}

1. Time to align the pedicle screw in anteroposterior and lateral views

2. Number of entry points made.

3. Number of exposures.

Putting the pedicle screw at the desired position

1. Slipping of the screw from the entry point.

2. Distance of the pedicle screw from the desired position at the entry, middle, and tip.

3. Breach of cortex during attempt of insertion of the screw.

4. Accuracy of measurement of the screw.

5. Time taken.

All these parameters were given weightage depending on the importance of the step. The trainee is scored according to these parameters with a maximum score of 100.

Weightage of marks for each step (50\% marks for each task)

1. Time to centering at the desired entry point (3 minutes given), then -1 mark is deducted for every 30 seconds extra taken ( $10 \%$ marks).

2. Number of exposure ( 5 allowed), then -1 for every extra exposure (20\% marks).
3. Improvement with each exposure, -1 for every deviation (20\% marks).

4. Slipping attempts ( -1 for each slip) (20\% marks).

5. Hole drilled with accuracy ( -1 for $0.5-\mathrm{mm}$ deviation from center of the hole) (5\% marks).

6. Screw inserted with accuracy ( $10 \%$ marks), -5 for every 5 -mm oversize/undersize of screw.

7. Time taken $20 \%$ marks ( 5 minutes allowed), -1 mark for every 30 seconds beyond 8 minutes. This time is subdivided into 2 tasks, 5 minutes for the first task and 3 minutes for the second task.

8. Cortical breach-points were deducted for cortical breach, 10 points for lateral breach, 20 points for medial breach, and 30 points for the anterior breach of the cortex.

The scoring was validated using senior trainees who perform this procedure on the training system and scoring them using our scoring system. There was a consistency in the scores achieved by the senior trainees when performing this procedure on the system. The scoring system was validated, and time was calculated after using the 6 trained orthopedic surgeons to perform the pedicle screws at the desired position and calculate their average timing to device the scores for each subset.

\section{RESULTS}

The results were analyzed using a statistical application SPSS version 22 and tested for the statistical difference in the improvement of the mental navigation of instruments after training with the computer-assisted orthopedic training system. The trainees were assessed based on the time taken along with the number of images acquired to complete the pedicle screw fixation and also the accuracy of the procedure. The assessors who assessed the results had no prior knowledge of the prior training of the residents and thus were blinded when evaluating the scores.

\section{Demographics}

Overall, 12 subjects (aged 27-37 years) who satisfied the inclusion criteria and agreed to participate were included in the study. There were 9 men and 3 women. All were junior orthopedic trainees.

\section{Scores}

There was a significant decrease in all the parameters in the first group (training) compared with the group 2 .

The number of exposures was 13.2 (mean) in the training group for pedicle screw insertion compared with 42.5 (mean) in the second group.

There was an overall decrease in all the parameters, and the mean score increased from 24.3 to 53.6 in the training 
group, and this was statistically significant using chi-square test $(\mathrm{p}=0.03)$.

There was a gradual improvement of the scores when the trainees were routinely practicing on the training system.

\section{DISCUSSION}

The article discusses the use of desktop-based simulation for the training of pedicle screw insertion in the lumbar spine by the junior orthopedic trainees. It also highlighted the improvement in their performance when they have used the training mode. The trainee while understanding the landmarks and the trajectory of the screws improved on all parameters. ${ }^{9}$ The scoring system was used to give formative feedback on their performance and improve on the weaker points of the trainee. This system helps trainee practice complex procedure on the desktop model without the need for C-arm and the costly setup required for the laboratorybased simulations. This is of utmost importance where more and more junior trainees are spending less time in the theater and have less exposure to hands-on training by senior surgeons in the theater. The system provides results and scores with which the surgeon and trainee can readily identify and improve on subtasks within the particular surgery.

The desktop version of the computer-assisted orthopedic training system would help trainee practice the procedure in a nonthreatening and more relaxing non-operating room environment. Thus, we wanted to create a simulator that could be used frequently and be accessible from a surgical trainee's own work environment, thus facilitating repetitive practice at times convenient to the trainee. ${ }^{14,15}$

The authors understand that the number of the subjects in our study is low, but it still demonstrates that our simulation system improves the accuracy and timing in junior orthopedic trainees. This study was conducted as a pilot study to see if desktop version of the computer-assisted orthopedic training system would help in training the junior orthopedic trainees in difficult procedures. Future studies will focus on increasing the number of trainees with repeated exposures to the training system to see if this would help them in sustaining their training.

The system in its present format can use both generic anatomical data and patient-specific data so that whole actual procedure can be simulated based on the patient images before it can be performed on the actual patient.

\section{CONCLUSION}

This article presents a desktop version of the computerassisted orthopedic training system. The participants in each of the group felt that the training system was helpful in training of complex procedures. The task analysis helps them in identifying their weaknesses in the subtasks, and the improvement in the scores in the training group supports the argument for this system to be used more routinely for training of junior orthopedic trainees for both simple and complex orthopedic procedures.

\section{ACKNOWLEDGMENT}

We thank all the junior and senior orthopedic trainees who have participated in this study.

\section{REFERENCES}

1. Rambani R, Viant W, Ward J, Mohsen A. Computerassisted orthopedic training system for fracture fixation. J Surg Educ. 2013;70(3):304-308.

2. Michelson JD. Simulation in orthopaedic education: an overview of theory and practice. J Bone Joint Surg Am. 2006;88(6):1405-1411.

3. Donohoe CL, Sayana MK, Kennedy MT, Niall DM. European working time directive: implications for surgical training. Ir Med J. 2010;103(2):57-59.

4. Andreatta PB, Hillard M, Krain LP. The impact of stress factors in simulation-based laparoscopic training. Surgery. 2010;147(5):631-639.

5. Blyth P, Stott NS, Anderson IA. A simulationbased training system for hip fracture fixation for use within the hospital environment. Injury. 2007;38(10): 1197-1203.

6. Van Herzeele I, O'Donoghue KG, Aggarwal R, Vermassen F, Darzi A, Cheshire NJ. Visuospatial and psychomotor aptitude predicts endovascular performance of inexperienced individuals on a virtual reality simulator. J Vasc Surg. 2010;51(4):1035-1042.

7. Fernandez JW, Mithraratne P, Thrupp SF, Tawhai $\mathrm{MH}$, Hunter PJ. Anatomically based geometric modelling of the musculo-skeletal system and other organs. Biomech Model Mechanobiol. 2004;2(3):139-155.

8. Bar-On E, Weigl DM, Becker T, Katz K, Konen O. Intraoperative $\mathrm{C}$-arm radiation affecting factors and reduction by an intervention program. J Pediatr Orthop. 2010;30(4):320-323.

9. Trantakis C, Dengl M, Grunert R, et al. Evaluation of a navigated and power controlled milling system "navigated control spine" for spinal surgery. Int $J$ Comput Assist Radiol Surg. 2009;4(5):487-495.

10. Bergeson RK, Schwend RM, DeLucia T, Silva SR, Smith JE, Avilucea FR. How accurately do novice 
surgeons place thoracic pedicle screws with the free hand technique? Spine. 2008;33(15):E501E507.

11. Rambani R, Varghese M. Computer assisted navigation in orthopaedics and trauma surgery. Orthop Trauma. 2014;28(1):50-57.

12. Malek S, Phillips R, Mohsen A, Viant W, Bielby M, Sherman K. Computer assisted orthopaedic surgical system for insertion of distal locking screws in intramedullary nails: a valid and reliable navigation system. Int J Med Robot. 2005;1(4):34-44.
13. Viant WJ, Phillips R, Griffiths JG, et al. A computer assisted orthopaedic surgical system for distal locking of intramedullary nails. Proc Inst Mech Eng H. 1997;211(4):293-300.

14. Tonetti J, Vadcard L, Girard P, Dubois M, Merloz P, Troccaz J. Assessment of a percutaneous iliosacral screw insertion simulator. Orthop Traumatol Surg Res. 2009;95(7):471-477.

15. Yehyawi TM, Thomas TP, Ohrt GT, et al. A simulation trainer for complex articular fracture surgery. J Bone Joint Surg Am. 2013;95(13):e92. 